\title{
Unstable splittings for real spectra
}

\author{
NitU KitChLOO \\ W STEPHEN WILSON
}

\begin{abstract}
We show that the unstable splittings of the spaces in the Omega spectra representing $B P, B P\langle n\rangle$ and $E(n)$ from [12] may be obtained for the real analogs of these spectra using techniques similar to those in [2]. Explicit calculations for $E R(2)$ are given.
\end{abstract}

\section{Introduction}

We are concerned with the $\mathbb{Z} /(2)$-equivariant (think complex conjugation) spaces (and their homotopy fixed points) associated with the $p=2$ spectra $B P, B P\langle n\rangle$ and $E(n)$. Recall that the homotopy of $B P$ is:

$$
B P_{*} \cong \mathbb{Z}_{(2)}\left[v_{1}, v_{2}, \ldots\right] \text { with degree } v_{n}=2\left(2^{n}-1\right) .
$$

Likewise,

$$
B P\langle n\rangle_{*} \cong \mathbb{Z}_{(2)}\left[v_{1}, v_{2}, \ldots, v_{n}\right] \text { and } E(n)_{*} \cong v_{n}^{-1} B P\langle n\rangle_{*} .
$$

We need some notation before we begin. A standard spectrum is just denoted $E$ and a real spectrum is denoted by $\mathbb{E}$. It is a bigraded (ie $R O(\mathbb{Z} /(2)$ )-graded) family of $\mathbb{Z} /(2)$-spaces $\underline{\mathbb{E}}_{(a, b)}$, where the indexing is given by $a+b \alpha$, where $\alpha$ is the sign representation in $R O(\mathbb{Z} /(2))$. The homotopy fixed-point space of the $\mathbb{Z} /(2)$-action on $\underline{\mathbb{E}}_{(a, b)}$ is denoted by $\underline{\mathbb{E}}(a, b)$. The collection $\underline{\mathbb{E}}_{(a, 0)}$ forms a $\mathbb{Z} /(2)$-equivariant Omega spectrum and $E R$ is the corresponding fixed-point spectrum with Omega spectrum $\underline{E R} a=\underline{\mathbb{E}}(a, 0)$.

In the second author's [12], the homotopy type of the spaces in the Omega spectrum for $B P$ was determined. A crucial step was showing that:

$$
\underline{B P}\langle n\rangle_{2\left(2^{n}-1\right)} \cong \underline{B P}\langle n\rangle_{4\left(2^{n}-1\right)} \times \underline{B P}\langle n-1\rangle_{2\left(2^{n}-1\right)} .
$$

For example,

$$
\underline{B P}_{0} \cong \prod_{k \geq 0} \underline{B P}\langle k\rangle_{2\left(2^{k}-1\right)}
$$


It follows that, for $m<2^{n}$,

$$
\underline{E}(n)_{2 m} \cong \underline{B P}\langle n\rangle_{2 m+2\left(2^{n}-1\right)} \times \overline{\prod_{k \geq 0}} \underline{B P}\langle n-1\rangle_{2 m-2 k\left(2^{n}-1\right)},
$$

where $\bar{\Pi}$ denotes the restricted product given by the colimit of finite products.

These results were all reproven using unstable operations in Boardman, Johnson and the second author's [1], but the easy direct proof was finally found by Boardman and the second author [2]. This last approach carries over to the bigraded equivariant case, and we obtain:

Theorem 1.2 There is an equivariant splitting of spaces:

$$
\underline{\mathbb{B} P}\langle n\rangle_{\left(2^{n}-1\right)(1+\alpha)} \cong \underline{\mathbb{B} P}\langle n\rangle_{2\left(2^{n}-1\right)(1+\alpha)} \times \underline{\mathbb{B} \mathbb{P}}\langle n-1\rangle_{\left(2^{n}-1\right)(1+\alpha)} .
$$

Theorem 1.3 There is an equivariant decomposition of $H$-spaces:

$$
\underline{\mathbb{P}}_{0} \cong \prod_{k \geq 0} \underline{\mathbb{B P}}\langle k\rangle_{\left(2^{k}-1\right)(1+\alpha)}
$$

Taking the homotopy fixed points, this gives a decomposition of the zeroth space in the Omega spectrum for $B P R$, the real $B P$.

Theorem 1.4 Let $m<2^{n}$, then there is an equivariant splitting:

$$
\underline{\mathbb{E}}(n)_{m(1+\alpha)} \cong \underline{\mathbb{B P}}\langle n\rangle_{\left(2^{n}-1+m\right)(1+\alpha)} \times \overline{\prod_{k \geq 0}} \underline{\mathbb{B P}}\langle n-1\rangle_{\left(m-k\left(2^{n}-1\right)\right)(1+\alpha)} .
$$

In the case of $n=2$, with period 6, of Equation (1.1), we get

$$
\underline{E}(2)_{0} \cong \underline{B P}\langle 2\rangle_{6} \times \overline{\prod_{k \geq 0}}\left(\mathbb{Z}_{(2)} \times B U_{(2)}\right) .
$$

In [3], Don Davis proves a major nonimmersion theorem for real projective spaces using the even part of the $B P\langle 2\rangle$ cohomology. All of his spaces are $v_{2}$-torsion free, so this is equivalent to using the theory $E(2)^{2 *}(-)$. Since his new information does not come from complex $K$-theory, it must be contained in the classifying spaces $\underline{B P}\langle 2\rangle_{2 k}$ for $k=1,2,3$.

Let $E R(n)$ be the homotopy fixed-point spectrum associated with $\mathbb{E}(n)$. Where $E(n)$ has periodicity $2\left(2^{n}-1\right), E R(n)$ has periodicity $2^{n+2}\left(2^{n}-1\right)$ (see our [7]). In particular, $E R(2)$ is 48 -periodic. 
$E R(2)^{16 *}(-)$ was used by us $[9 ; 10]$ to improve some of Davis's nonimmersions slightly. The result analogous to Equation (1.5) from the splitting of Theorem 1.4 gives, for a space $Y 0$, the homotopy fixed points of $\underline{\mathbb{B P}}\langle 2\rangle_{3(1+\alpha)}$ :

$$
\underline{E R}(2)_{0} \cong Y 0 \times \overline{\prod_{k \geq 0}}\left(\mathbb{Z}_{(2)} \times B O_{(2)}\right) .
$$

The space $Y 0$ has lowest homotopy in degree 3 . We see below that it splits as the product of two spaces. It is beyond what we do in this paper, but this split is as the product of a space $Y 0^{\prime}$ with lowest homotopy degree 6 , and the space that you get by killing 2 times the generator of $H^{4}$ (BSpin).

We have one last splitting:

Theorem 1.6 Let $m<2^{n}$, there is an equivariant equivalence

$$
\begin{aligned}
\Omega^{2^{n+2}\left(2^{n-1}-1\right)} \underline{\mathbb{B}}\langle n\rangle_{\left(2^{n}-2+m\right)(1+\alpha)} & \\
& \cong \underline{\mathbb{B P}}\langle n\rangle_{\left(2^{n}-1+m\right)(1+\alpha)} \times \prod_{0 \leq k \leq 2^{n}-2} \underline{\mathbb{B P}}\langle n-1\rangle_{\left(m-k\left(2^{n}-1\right)\right)(1+\alpha)} .
\end{aligned}
$$

Running through this from $m=1$ to $m=2^{n}-1$ goes through a multiple of the complete periodicity.

In the case of the homotopy fixed points for the $n=2$ case with 48-periodicity, the theorem gives us spaces $Y 1$ and $Y 2$, the homotopy fixed points for $\underline{\mathbb{B}}\langle 2\rangle_{4(1+\alpha)}$ and $\underline{\mathbb{P}}\langle 2\rangle_{2(1+\alpha)}$ respectively, such that:

$$
\Omega^{16} Y 0 \cong Y 1 \times B O \times\left(\mathbb{Z}_{(2)} \times B O\right) \times\left(\mathbb{Z}_{(2)} \times B O\right),
$$

where $Y 1$ has lowest degree element in degree 4. We also have

$$
\Omega^{16} Y 1 \cong Y 2 \times\left(\mathbb{Z}_{(2)} \times B O\right) \times\left(\mathbb{Z}_{(2)} \times B O\right),
$$

where the lowest degree element in $Y 2$ is in degree 2 and

$$
Y 2 \cong Y 2^{\prime} \times B S O,
$$

where $Y 2^{\prime}$ is the homotopy fixed points for $\underline{\mathbb{P}}\langle 2\rangle_{5(1+\alpha)}$ with lowest degree homotopy in degree 5. Finally,

$$
\Omega^{16} Y 2 \cong Y 0 \times\left(\mathbb{Z}_{(2)} \times B O\right) \times\left(\mathbb{Z}_{(2)} \times B O\right) \times\left(\mathbb{Z}_{(2)} \times B O\right) .
$$

All put together we have:

$$
\Omega^{48} Y 0 \cong Y 0 \times \prod_{k=1}^{8}\left(\mathbb{Z}_{(2)} \times B O\right) .
$$


This begs the question, what are the splittings for $\Omega^{8} Y 0, \Omega^{8} Y 1$, and $\Omega^{8} Y 2$ ? Although our description of the homotopy of $Y 0$ suggests a conjecture, we do not pursue this here.

Since our new nonimmersion results certainly don't come from $K$-theory, the new information is contained in the spaces $Y 0, Y 1$ and $Y 2$.

In [8], we computed the homology of the spaces $\underline{E R}(n)_{2^{n+2} k}$ and all of the homotopy fixed-point spaces in all of the splitting theorems listed so far. In the case of $E R(2)$ we went further and computed the homology of all 48 spaces in the Omega spectrum. We can read off the homology of $Y 0 \times Y 1 \times Y 2$ with ease from that computation and do so in Section 7.

The homotopy of ER(2) is computed in Hu and Kriz [6] and our [7], but best described for our purposes in [8, Proposition 2.1]. We reorganize the description of this homotopy so the homotopy of all the $B O$ is visible, even as the spaces are looped down. There is very little "core" homotopy left that never shows up in a $B O$, and this is completely described in Section 6.

It is worth pointing out that there is compelling evidence that suggests that our spectrum $E R(2)$ is equivalent to the spectrum TM(3) (topological modular forms with a level 3 structure) constructed by Mahowald and Rezk [11]. In particular, the unstable splittings studied in our paper may have interesting geometric content.

Before we begin with the actual technical results, we mention a word about our notation. We have chosen to be consistent with [6] in our notation. Consequently, in the sequel $\alpha$ will denote the sign representation of $\mathbb{Z} /(2)$, and $\sigma$ will denote the "shift" operator that suspends a spectrum by the virtual representation $\alpha-1$. Unfortunately, this notation is in unavoidable conflict with Hill and Hopkins's [4], where $\sigma$ is used to denote the sign representation.

The organization of the paper is as follows. We first prove the main technical result we need. Then we have three sections proving all the splitting theorems. Section 6 describes the homotopy and Section 7 the homology of interest. Finally, there is a brief appendix proving some results in a form that we need for the main technical result.

\section{Proof of the main technical theorem}

Definition 2.1 [8] A $\mathbb{Z} /(2)$-space $X$ is said to be projective if:

(1) $H_{*}(X ; \mathbb{Z})$ is of finite type.

(2) $X$ is homeomorphic to $\bigvee_{I}\left(\mathbb{C} P^{\infty}\right)^{\wedge k_{I}}$ for some weakly increasing sequence of integers $k_{I}$, with the $\mathbb{Z} /(2)$-action given by complex conjugation. 
Definition 2.2 A $\mathbb{Z} /(2)$-equivariant $H$-space $Y$ is said to have the projective property if there exists a projective space $X$, along with a $\mathbb{Z} /(2)$-equivariant map $f: X \rightarrow Y$, such that $H_{*}\left(Y ; \mathbb{Z}_{(2)}\right)$ is generated as an algebra by the image of $f$.

Spaces with the projective property are not rare because many spaces have homology generated by the image of elements coming from complex projective space. Our examples include $\mathbb{M U}_{k(1+\alpha)}, \mathbb{B P}_{k(1+\alpha)}$ and $\mathbb{B} \mathbb{P}\langle n\rangle_{k(1+\alpha)}$, where this last is only for $k<2^{n+1}$. For these diagonal spaces (as part of a bigraded real spectrum), we have the following theorem:

Theorem 2.3 Let $Y$ be a space with the projective property. Given any integer $n \geq 0$, let $\mathbb{E}$ be the $\mathbb{M U}$-module spectrum $\mathbb{B P}$ or $\mathbb{B} \mathbb{P}\langle n\rangle$. Then the following map is surjective:

$$
\mathbb{M} \mathbb{U}_{(2)}^{a+b \alpha}(Y) \longrightarrow \mathbb{E}^{a+b \alpha}(Y), \quad a \leq b<2^{n+1},
$$

where $\mathbb{M U}(2)$ denotes the 2-localization of the spectrum.

The above theorem allows us to prove equivariant versions of all classical splitting results one has for spaces (with the projective property) that appear in the Omega spectra representing $\mathbb{B} \mathbb{P}, \mathbb{B} \mathbb{P}\langle n\rangle$ and $\mathbb{E}(n)$.

Proof By [6], $\mathbb{B P}$ is an equivariant retract of $\mathbb{M U}_{(2)}$. So we only need to establish the theorem for $\mathbb{E}=\mathbb{B P}\langle n\rangle$. Furthermore, from Theorem A.3 in the appendix, we may replace $\mathbb{E}$ by the completion $\widehat{\mathbb{E}}=\operatorname{Map}\left(E \mathbb{Z} /(2)_{+}, \mathbb{E}\right)$ in the degrees we are interested in.

Now recall that by definition of the projective property, there is a projective space $X$ so that there is a $\mathbb{Z} /(2)$-equivariant map $f: X \rightarrow Y$, whose image generates the homology. It follows that $H_{*}\left(Y, \mathbb{Z}_{(2)}\right)$ is free, and the $\mathbb{Z} /(2)$-equivariant map $\Omega \Sigma X \rightarrow Y$ is surjective in homology. The Atiyah-Hirzebruch spectral sequence now shows that $M U_{*}(\Omega \Sigma X)$ and $M U_{*}(Y)$ are free $M U_{*}$-modules, and the map $M U_{*}(\Omega \Sigma X) \rightarrow M U_{*}(Y)$ is split surjective. The next step is to pick equivariant representatives for the splitting.

Let $Z$ denote the $\mathbb{Z} /(2)-\mathrm{CW}$ complex $\Omega \Sigma X$. Now $Z$ admits the equivariant James filtration, which is known to split (equivariantly) into the wedge of spectra of the form $X^{\wedge k}$. Now consider the spectral sequence constructed using the cellular filtration of $Z$ induced by the canonical (equivariant) cellular filtration of the projective space $X$, and converging to $\mathbb{M} \mathbb{U}_{*, *}(Z)$. Since the James filtration of $Z$ splits equivariantly, and $X$ is projective, all generators in the $E_{2}$-term above represent permanent cycles, and therefore the above spectral sequence collapses. It follows that $\mathbb{M} \mathbb{U}_{*, *}(Z)$ is 
a free $\mathbb{M U}_{*, *}$-module, or equivalently, $\mathbb{M U} \wedge Z$ is a free $\mathbb{M U}$-module spectrum on a generating set of finite type: $\mathbb{M} \mathbb{U} \wedge Z=\bigvee_{i} \Sigma^{k_{i}(1+\alpha)} \mathbb{M U}$, where $\left\{k_{1}, k_{2}, \ldots\right\}$ is a weakly increasing sequence of nonnegative integers. We may pick a suitable subsequence $\left\{\beta_{1}, \beta_{2}, \ldots\right\}$ in $\left\{k_{1}, k_{2}, \ldots\right\}$ so that we get a $\mathbb{Z} /(2)$-equivariant map

$$
\bigvee \Sigma^{\beta_{i}(1+\alpha)} \mathbb{M U} \longrightarrow Y \wedge \mathbb{M} \mathbb{U}
$$

which is a (nonequivariant) equivalence. On freeing up our spectra, it follows that we have an equivariant equivalence of $\mathbb{M} \mathbb{U}$-module spectra:

$$
\bigvee \Sigma^{\beta_{i}(1+\alpha)} \wedge E \mathbb{Z} /(2)_{+} \wedge \mathbb{M U} \longrightarrow E \mathbb{Z} /(2)_{+} \wedge Y \wedge \mathbb{M} \mathbb{U}
$$

Now let $n \geq 0$ be any integer, and let $\mathbb{E}$ be the $\mathbb{M U}$-module spectrum $\mathbb{M U}(2)$ or $\mathbb{B P}\langle n\rangle$. Mapping out of the above equivalence in the category of $\mathbb{M} \mathbb{U}$-module spectra, we observe that in degrees $a+b \alpha$ for $a \leq b<2^{n+1}$ we have

$$
\mathbb{E}^{*, *}(Y)=\widehat{\mathbb{E}}^{*, *}(Y)=\mathbb{E}^{*, *}\left\langle\left\langle\gamma_{1}, \gamma_{2}, \ldots\right\rangle\right\rangle,
$$

where $\gamma_{i}$ is the generator in degree $\beta_{i}(1+\alpha)$. In particular, it follows that $\mathbb{M U}_{(2)}^{a+b \alpha}(Y)$ surjects onto $\mathbb{E}_{(2)}^{a+b \alpha}(Y)$ for $a \leq b<2^{n+1}$.

Remark 2.4 Let $\mathbb{E}(n)=\mathbb{B} \mathbb{P}\langle n\rangle\left[v_{n}^{-1}\right]$ be the equivariant Johnson-Wilson spectrum. Then the above proof also shows that the map

$$
\mathbb{M U}_{(2)}\left[v_{n}^{-1}\right]^{*, *}(Y) \longrightarrow \mathbb{E}(n)^{*, *}(Y)
$$

is surjective in all bidegrees. From this it follows easily that $\mathbb{E}(n)$ splits off unstably from the equivariant $E$-infinity ring spectrum $\mathbb{M U}_{(2)}\left[v_{n}^{-1}\right]$.

\section{Splitting: $\underline{\mathbb{B P}}\langle n\rangle_{\left(2^{n}-1\right)(1+\alpha)}$}

We will use the main theorem of the previous section to construct unstable splittings of various spaces that have the projective property. In this section we prove Theorem 1.2.

Proof of Theorem 1.2 Consider the equivariant fibration:

$$
\underline{\mathbb{B P}} n_{2\left(2^{n}-1\right)(1+\alpha)} \stackrel{v_{n}}{\longrightarrow} \underline{\mathbb{B P}}\langle n\rangle_{\left(2^{n}-1\right)(1+\alpha)} \longrightarrow \underline{\mathbb{B P}}\langle n-1\rangle_{\left(2^{n}-1\right)(1+\alpha)} .
$$

Since $\mathbb{B P}\langle n-1\rangle_{\left(2^{n}-1\right)(1+\alpha)}$ is a space with the projective property, we know from the main theorem that the following map is surjective:

$\mathbb{B} \mathbb{P}\langle n\rangle^{\left(2^{n}-1\right)(1+\alpha)}\left(\underline{\mathbb{B} \mathbb{P}}\langle n-1\rangle_{\left(2^{n}-1\right)(1+\alpha)}\right)$

$$
\longrightarrow \mathbb{B P}\langle n-1\rangle^{\left(2^{n}-1\right)(1+\alpha)}\left(\underline{\mathbb{B P}}\langle n-1\rangle_{\left(2^{n}-1\right)(1+\alpha)}\right) \text {. }
$$


This implies that there is a section $\tau: \underline{\mathbb{B} P}\langle n-1\rangle_{\left(2^{n}-1\right)(1+\alpha)} \rightarrow \underline{\mathbb{P}}\langle n\rangle_{\left(2^{n}-1\right)(1+\alpha)}$ lifting the identity map on $\underline{\mathbb{P}}\langle n-1\rangle_{\left(2^{n}-1\right)(1+\alpha)}$ inducing a splitting of the form we wanted.

Remark 3.1 Notice that the above splitting is not as $H$-spaces. However, for all nontrivial loopings of the form $(r+s \alpha)$ for $r, s \geq 0$, this splitting yields splittings as $H$-spaces.

\section{The case of $\underline{\mathbb{B P}} 0$}

Let us fix splittings as $H$-spaces,

$$
\underline{\mathbb{B} P}\langle n\rangle_{0} \cong \underline{\mathbb{B} \mathbb{P}}\langle n\rangle_{\left(2^{n}-1\right)(1+\alpha)} \times \underline{\mathbb{B} \mathbb{P}}\langle n-1\rangle_{0},
$$

that were constructed in the previous section. We can now prove Theorem 1.3 from the introduction.

Proof of Theorem 1.3 Consider commutative diagrams of the form:

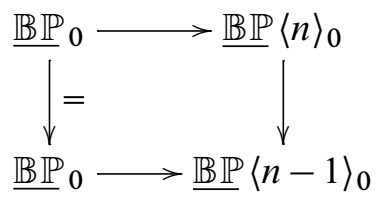

Notice that the horizontal maps get increasingly connective as $n$ increases. In addition, the right vertical maps split by the previous section. It follows on taking homotopy inverse limits that one has a decomposition of $H$-spaces:

$$
\underline{\mathbb{B} P} 0 \cong \prod_{k \geq 0} \underline{\mathbb{B P}}\langle k\rangle_{\left(2^{k}-1\right)(1+\alpha)} .
$$

\section{The case of $\underline{\mathbb{E}}(n)_{m(1+\alpha)}$}

In this section we prove Theorems 1.4 and 1.6 from the introduction.

Proof of Theorem 1.4 Consider the commutative diagram with a split top horizontal sequence:

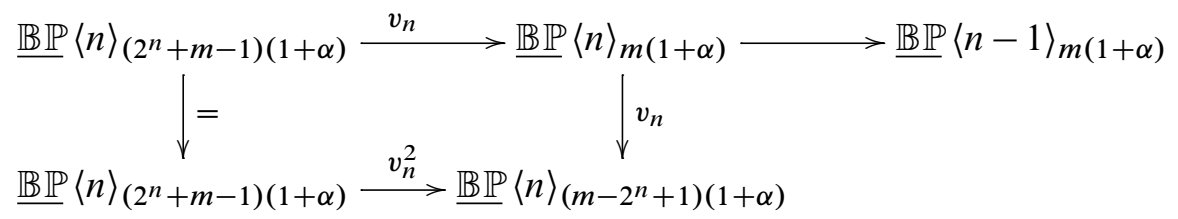


Notice also that the vertical map given by multiplication by $v_{n}$ splits with cokernel given by $\mathbb{B} \mathbb{P}\langle n-1\rangle_{\left(m-2^{n}+1\right)(1+\alpha)}$. It follows that the bottom horizontal map is also split with cokernel given by $\underline{\mathbb{B}}\langle n-1\rangle_{m(1+\alpha)} \times \underline{\mathbb{B}}\langle n-1\rangle_{\left(m-2^{n}+1\right)(1+\alpha)}$.

Continuing the diagram vertically, with increasing powers of $v_{n}$, and taking colimits, we therefore have an equivariant splitting:

$$
\underline{\mathbb{E}}(n)_{m(1+\alpha)} \cong \underline{\mathbb{B} \mathbb{P}}\langle n\rangle_{\left(2^{n}+m-1\right)(1+\alpha)} \times \overline{\prod_{k \geq 0}} \underline{\mathbb{B} P}\langle n-1\rangle_{\left(m-k\left(2^{n}-1\right)\right)(1+\alpha)} .
$$

As noted earlier, for $m=2^{n}-1$, this splitting is not as $H$-spaces. However, all nontrivial loops on that splitting do yield a splitting as $H$-spaces.

The proof of Theorem 1.4 lets us identify the splitting in terms of homotopy. In particular, the proof shows that the following map given by multiplication by $v_{n}^{i}$ is split:

$$
\begin{aligned}
\underline{\mathbb{B}}\langle n\rangle_{\left(2^{n}+m-1\right)(1+\alpha)} \longrightarrow \underline{\mathbb{B}}\langle n\rangle_{\left(m-i\left(2^{n}-1\right)\right)(1+\alpha)} & \\
& \longrightarrow \prod_{0 \leq k \leq i} \underline{\mathbb{B}}\langle n-1\rangle_{\left(m-k\left(2^{n}-1\right)\right)(1+\alpha)} .
\end{aligned}
$$

In addition, the image

$$
\pi_{*} \underline{\mathbb{B} \mathbb{P}}\langle n\rangle_{\left(m-i\left(2^{n}-1\right)\right)(1+\alpha)} \longrightarrow \pi_{*} \underline{\mathbb{E}}(n)_{m(1+\alpha)}
$$

is exactly

$$
v_{n}^{-i} \pi_{*} \mathbb{B} \mathbb{P}\langle n\rangle \cap \pi_{*} \underline{\mathbb{E}}(n)_{m(1+\alpha)} .
$$

It follows that:

Theorem 5.2 Under the splitting given in the previous theorem, the image

$$
\pi_{*}\left(\underline{\mathbb{B} \mathbb{P}}\langle n\rangle_{\left(2^{n}+m-1\right)(1+\alpha)} \times \prod_{0 \leq k \leq i} \underline{\mathbb{B} \mathbb{P}}\langle n-1\rangle_{\left(m-k\left(2^{n}-1\right)\right)(1+\alpha)}\right) \longrightarrow \pi_{*} \underline{\mathbb{E}}(n)_{m(1+\alpha)}
$$

is exactly

$$
v_{n}^{-i} \pi_{*} \mathbb{B} \mathbb{P}\langle n\rangle \cap \pi_{*} \underline{\mathbb{E}}(n)_{m(1+\alpha)} .
$$

In particular, $\pi_{*} \underline{\mathbb{B} \mathbb{P}}\langle n-1\rangle_{\left(m-i\left(2^{n}-1\right)\right)(1+\alpha)}$ is supported on elements in $\pi_{*} \underline{\mathbb{E}}(n)_{m(1+\alpha)}$ with $v_{n}$-exponent exactly $-i$.

Having identified the splitting in homotopy, we can analyze the periodicity of the space $\underline{\mathbb{E}}(n)_{0}$. For this, let $\lambda=2^{2 n+1}-2^{n+2}+1$, and recall that there is an invertible element $\bar{y}(n) \in \pi_{\lambda-1} \underline{\mathbb{E}}(n)_{-(1+\alpha)}$ [7]. This element is given by $y(n)=v_{n}^{2^{n}-1} \sigma^{-2^{n+1}\left(2^{n-1}-1\right)}$. An equivariant equivalence $\underline{\mathbb{E}}(n)_{m(1+\alpha)} \rightarrow \Omega^{\lambda-1} \underline{\mathbb{E}}(n)_{(m-1)(1+\alpha)}$ is obtained from 
multiplication by $y(n)$. Since the $v_{n}$ exponent of $y(n)$ is exactly $2^{n}-1$, we derive the useful consequence of the above theorem, namely we have proven Theorem 1.6 of the introduction.

\section{The homotopy of $E R(2)$}

In the computation of the homotopy of $E R(2)$ by Hu and Kriz [6] and ourselves [7], the only concern was for the degree mod 48 , but that no longer suffices. The homotopy of the fixed-point spaces for the bigraded object is generated by:
$v_{0}(1)$ in degree $2 \alpha-2$,
$v_{0}(0)=2$ in degree 0 ,
$v_{2}$ in degree $3 \alpha+3$,
$v_{0}(2)$ in degree $4 \alpha-4$,
$v_{1}(0)$ in degree $\alpha+1$,
$a$ in degree $-\alpha$,
$v_{0}(3)$ in degree $6 \alpha-6$,
$v_{1}(1)$ in degree $5 \alpha-3$,
$\sigma^{8}$ in degree $8 \alpha-8$.

Both $v_{2}$ and $\sigma^{8}$ are invertible and we define the invertible:

$$
y(2)=y=v_{2}^{3} \sigma^{-8} \quad \text { in degree } 17+\alpha .
$$

To get the homotopy of $E R(2)$ we need the $\alpha$-coordinate equal to zero. We can move our generators there using $y$ and rename them in the process. We capture their degrees doing this:

$$
\begin{array}{rlrl}
y^{-2} v_{0}(1) & =\alpha_{1} \text { in degree }-36, & y^{-1} v_{1}(0) & =\alpha \text { in degree }-16, \\
y^{-4} v_{0}(2) & =\alpha_{2} \text { in degree }-72, & y^{-5} v_{1}(1) & =w \text { in degree }-88, \\
y^{-6} v_{0}(3) & =\alpha_{3} \text { in degree }-108, & y^{-3} v_{2} & =g \text { in degree }-48, \\
y a & =x \text { in degree } 17 .
\end{array}
$$

The element $g$ is our periodicity operator. Apologies for using $\alpha$ for two different things.

The relations as written down in [8] must be modified to take into account the real degrees as opposed to just the mod 48 degrees. The relations are given by:

$$
\begin{aligned}
& 0=2 x=x^{7}=x^{3} w=x^{3} \alpha=x \alpha_{i}, \quad w \alpha_{2}=2 \alpha g^{3}, \quad \alpha^{2} g^{3}=w^{2}, \\
& \alpha_{1}^{2}=2 \alpha_{2}, \quad \alpha_{2}^{2}=4 g^{3}, \quad \alpha_{3}^{2}=2 \alpha_{2} g^{3}, \\
& \alpha_{1} \alpha_{2}=2 \alpha_{3}, \quad \alpha_{1} \alpha_{3}=4 g^{3}, \quad \alpha_{2} \alpha_{3}=2 \alpha_{1} g^{3}, \\
& \alpha \alpha_{1} g^{3}=\alpha_{3} w, \quad \alpha \alpha_{2}=2 w, \quad \alpha \alpha_{3}=\alpha_{1} w \text {. }
\end{aligned}
$$


As a module over $\mathbb{Z}_{(2)}\left[\alpha, g, g^{-1}\right]$, the homotopy can be described as having generators

$$
1, \quad w, \quad \alpha_{1}, \quad \alpha_{3} \text { and } \alpha_{2},
$$

with one relation

$$
\alpha \alpha_{2}=2 w,
$$

copies of $\mathbb{Z} /(2)\left[\alpha, g, g^{-1}\right]$ on generators

$$
x, \quad x^{2}, \quad x w, \quad x^{2} w
$$

and copies of $\mathbb{Z} /(2)\left[g, g^{-1}\right]$ on

$$
x^{3}, \quad x^{4}, \quad x^{5}, \quad x^{6} .
$$

We want to rewrite this using a special element, $h=g^{-1} \alpha^{3}$, of degree 0 . Because $g$ is invertible, we can replace any $\alpha^{3}$ with $h$. Rewritten using $h$, we have that the homotopy of $E R(2)$ is a $\mathbb{Z}_{(2)}$ on each of

$$
\alpha^{j} w^{\epsilon} g^{s} h^{k}, \quad \alpha_{1} \alpha^{j} g^{s} h^{k}, \quad \alpha_{3} \alpha^{j} g^{s} h^{k}, \quad \alpha_{2} g^{s},
$$

where $0 \leq j \leq 2,0 \leq \epsilon \leq 1, s \in \mathbb{Z}, k \geq 0$, and a $\mathbb{Z} /(2)$ on each of

$$
x^{i} \alpha^{j} w^{\epsilon} g^{s} h^{k}, \quad 1 \leq i \leq 2 \text { and } x^{v} g^{s}, \quad 3 \leq v \leq 6,
$$

where $j, \epsilon, s$ and $k$ are as above.

It is easy to see (look mod 48) that the elements of degree zero are just the $h^{k}$, but we can do much better and write down all elements of nonnegative degrees. Let $s, k \geq 0$, the elements and the degrees of all nonnegative degree elements are as follows:

\begin{tabular}{lrrr} 
deg & \multicolumn{3}{l}{ deg } \\
$48 s$ & $g^{-s} h^{k}$ & $48 s+24$ & $w \alpha^{2} g^{-s-3} h^{k}$ \\
$48 s+1$ & $x \alpha g^{-s} h^{k}$ & $48 s+25$ & $x w g^{-s-2} h^{k}$ \\
$48 s+2$ & $x^{2} \alpha^{2} g^{-s} h^{k}$ & $48 s+26$ & $x^{2} \alpha w g^{-s-2} h^{k}$ \\
$48 s+4$ & $\alpha_{3} \alpha^{2} g^{-s-3} h^{k}$ & $48 s+28$ & $\alpha_{1} \alpha^{2} g^{-s-2} h^{k}$ \\
$48 s+8$ & $w g^{-s-2} h^{k}$ & $48 s+32$ & $\alpha g^{-s-1} h^{k}$ \\
$48 s+9$ & $x w \alpha g^{-s-2} h^{k}$ & $48 s+33$ & $x \alpha^{2} g^{-s-1} h^{k}$ \\
$48 s+10$ & $x^{2} w \alpha^{2} g^{-s-2} h^{k}$ & $48 s+34$ & $x^{2} g^{-s} h^{k}$ \\
$48 s+12$ & $\alpha_{1} g^{-s-1} h^{k}$ & $48 s+36$ & $\alpha_{3} g^{-s-3} h^{k}$ \\
$48 s+16$ & $\alpha^{2} g^{-s-1} h^{k}$ & $48 s+40$ & $w \alpha g^{-s-3} h^{k}$ \\
$48 s+17$ & $x g^{-s} h^{k}$ & $48 s+41$ & $x w \alpha^{2} g^{-s-3} h^{k}$ \\
$48 s+18$ & $x^{2} \alpha g^{-s} h^{k}$ & $48 s+42$ & $x^{2} w g^{-s-2} h^{k}$ \\
$48 s+20$ & $\alpha_{3} \alpha g^{-s-3} h^{k}$ & $48 s+44$ & $\alpha_{1} \alpha g^{-s-2} h^{k}$
\end{tabular}


There are also elements we call CORE homotopy (still with $s \geq 0$ ):

$$
\begin{array}{lr}
48 s+3 & g^{1-s} x^{3} \\
48 s+6 & g^{2-s} x^{6} \\
48 s+20 & g^{1-s} x^{4} \\
48 s+24 & g^{-s-2} \alpha_{2} \\
48 s+37 & g^{1-s} x^{5}
\end{array}
$$

We will also need elements we call $\mathrm{CORE}^{+}$, where we have the same as the CORE elements except that $s \in \mathbb{Z}$.

We note that $h$ times the elements with $x^{3}$ in them are all zero because of the relation $x^{3} \alpha=0$, but that

$$
g^{-s-2} \alpha_{2} h=2 w \alpha^{2} g^{-s-3} .
$$

Note also that the non-CORE homotopy is exactly the same as an infinite number of copies of $\mathbb{Z}_{(2)} \times B O$. Unfortunately, that isn't how it works.

Going back to the splitting in the $n=2$ case, we have:

$$
\underline{\mathbb{E}}(2)_{0} \cong \underline{\mathbb{B P}}\langle 2\rangle_{3(1+\alpha)} \times \overline{\prod_{k \geq 0}} \underline{\mathbb{B P}}\langle 1\rangle_{-k 3(1+\alpha)}
$$

First, we note that the homotopy fixed points of $\underline{\mathbb{E}}(2)_{0}$ is $\underline{E R}(2)_{0}$, the zeroth space of the Omega spectrum for $E R(2)$. Next, we note that the homotopy fixed points of $\underline{\mathbb{B} P}\langle 1\rangle_{-k 3(1+\alpha)}$ is just $\mathbb{Z}_{(2)} \times B O$.

Letting $Y 0$ be the homotopy fixed points for $\underline{\mathbb{B} \mathbb{P}}\langle 2\rangle_{3(1+\alpha)}$, we have, as in the introduction:

$$
\underline{E R}(2)_{0} \cong Y 0 \times \overline{\prod_{k \geq 0}}\left(\mathbb{Z}_{(2)} \times B O\right) .
$$

The space $Y 0$ is of particular interest and so we would like to have its homotopy. We already know its homology from [8], so we know it is 2-connected, so the bottom homotopy group is a $\mathbb{Z} /(2)$ in degree 3 generated by $g x^{3}$.

Each $h^{k}, k \geq 0$, must be the generator for a $\mathbb{Z}_{(2)}$ associated with one of the $\mathbb{Z}_{(2)} \times B O$. It is now clear that if we know the homotopy of the $\mathbb{Z}_{(2)} \times B O$ associated with 1 (ie $k=0$ above), we get the homotopy of all the others by multiplying by powers of $h$. Since $Y 0$ has no homotopy in degree 1 , the $\mathbb{Z} /(2)$ in degree 1 for our $\mathbb{Z}_{(2)} \times B O$ associated with 1 must be $x \alpha$. Likewise for the 2-degree element $x^{2} \alpha^{2}$. The 4-degree 
element is a $\mathbb{Z}_{(2)}$ on $\alpha_{3} \alpha^{2} g^{-3}$. From this we can compute our 8 -degree homotopy element by squaring:

$$
\left(\alpha_{3} \alpha^{2} g^{-3}\right)^{2}=\alpha_{3}^{2} \alpha^{4} g^{-6}=2 \alpha_{2} g^{3} \alpha^{4} g^{-6}=4 w \alpha^{3} g^{-3}=4 w g^{-2} h .
$$

The degree 8 element in our first $B O$ is $w g^{-2} h$. This is our $B O$ periodicity element. From this we can now find all of the rest of the homotopy of our first $B O$. The main thing left to do is compute powers of the periodicity element.

For degree 16 we have

$$
\left(w g^{-2} h\right)^{2}=w^{2} g^{-4} h^{2}=\alpha^{2} g^{3} g^{-4} h^{2}=\alpha^{2} g^{-1} h^{2} .
$$

Continuing in this fashion: in degree 24 we have $w \alpha^{2} g^{-3} h^{3}$; degree $32, \alpha g^{-1} h^{5}$; degree $40, w \alpha g^{-3} h^{6}$, degree $48, g^{-1} h^{8}$.

This sequence had a nice ending. Multiplication by the periodicity element $g$ corresponds to looping down 48 times and takes this 48 -degree element to $h^{8}$ in the 9-th copy of $\mathbb{Z}_{(2)} \times B O$.

We can now write down all the homotopy generators of our first $\mathbb{Z}_{(2)} \times B O$, for $k \geq 0$ :

\begin{tabular}{lrrr} 
deg & \multicolumn{3}{l}{ deg } \\
$48 k$ & $g^{-k} h^{8 k}$ & $48 k+24$ & $w \alpha^{2} g^{-k-3} h^{8 k+3}$ \\
$48 k+1$ & $x \alpha g^{-k} h^{8 k}$ & $48 k+25$ & $x w g^{-k-2} h^{8 k+4}$ \\
$48 k+2$ & $x^{2} \alpha^{2} g^{-k} h^{8 k}$ & $48 k+26$ & $x^{2} \alpha w g^{-k-2} h^{8 k+4}$ \\
$48 k+4$ & $\alpha_{3} \alpha^{2} g^{-k-3} h^{8 k}$ & $48 k+28$ & $\alpha_{1} \alpha^{2} g^{-k-2} h^{8 k+4}$ \\
$48 k+8$ & $w g^{-k-2} h^{8 k+1}$ & $48 k+32$ & $\alpha g^{-k-1} h^{8 k+5}$ \\
$48 k+9$ & $x w \alpha g^{-k-2} h^{8 k+1}$ & $48 k+33$ & $x \alpha^{2} g^{-k-1} h^{8 k+5}$ \\
$48 k+10$ & $x^{2} w \alpha^{2} g^{-k-2} h^{8 k+1}$ & $48 k+34$ & $x^{2} g^{-k} h^{8 k+6}$ \\
$48 k+12$ & $\alpha_{1} g^{-k-1} h^{8 k+2}$ & $48 k+36$ & $\alpha_{3} g^{-k-3} h^{8 k+6}$ \\
$48 k+16$ & $\alpha^{2} g^{-k-1} h^{8 k+2}$ & $48 k+40$ & $w \alpha g^{-k-3} h^{8 k+6}$ \\
$48 k+17$ & $x g^{-k} h^{8 k+3}$ & $48 k+41$ & $x w \alpha^{2} g^{-k-3} h^{8 k+6}$ \\
$48 k+18$ & $x^{2} \alpha g^{-k} h^{8 k+3}$ & $48 k+42$ & $x^{2} w g^{-k-2} h^{8 k+7}$ \\
$48 k+20$ & $\alpha_{3} \alpha g^{-k-3} h^{8 k+3}$ & $48 k+44$ & $\alpha_{1} \alpha g^{-k-2} h^{8 k+7}$
\end{tabular}

The homotopy of all of the other $\mathbb{Z}_{(2)} \times B O$ are obtained by taking powers of $h$ times this.

What remains must be the homotopy of $Y 0$. This is the CORE and all of the above for lower powers of $h$ than what is used. This has a nice $B O$-related description. Recall the notation $b o\langle n\rangle$ for the spectrum obtained from the connective version of 
$\mathbb{Z}_{(2)} \times B O$ by killing off all of the stable homotopy groups in degrees less than $n$. The stable homotopy of $b o=b o\langle 0\rangle$ is the same as the unstable homotopy of $\mathbb{Z}_{(2)} \times B O$.

We can now read off a description of the homotopy of the zeroth space of $E R(2)$ from the above. We have already written down the homotopy for all of the copies of $\mathbb{Z}_{(2)} \times B O$, so all we need now is the homotopy of $Y 0$. We first take every element that is divisible by $h^{8 k+1}$ and we see, using Bott periodicity and the degree of $g$, that there is an isomorphism

$$
\pi_{*} b o\langle 48 k+8\rangle \cong \Sigma^{48 k} \pi_{*} b o\langle 8\rangle \cong g^{-k_{*}} \pi_{*} b\langle 8\rangle
$$

and, for the $k=0$ case, $h$ multiplied times $\pi_{*} b o\langle 8\rangle$ maps this homotopy injectively into our first: $\mathbb{Z}_{(2)} \times B O$. The bottom class of $\pi_{*} b o\langle 8\rangle$ in degree 8 is $w g^{-2}$ and maps by $h$ to the 8 degree class $w g^{-2} h$ in that first $\mathbb{Z}_{(2)} \times B O$ listed above. Furthermore, the 48-degree homotopy would be $g^{-1} h^{7}$ (we divide all the higher terms by one $h$ ), so looping 48 times, ie multiplying by $g$, gives us $h^{7}$, and we see that the homotopy we are looking at in this instance becomes the homotopy of the $\mathbb{Z}_{(2)} \times B O$ associated with $h^{7}$.

Continuing, dividing by $h^{8 k+i}, 0<i \leq 8$, and using the same notation where $g^{-k}$ keeps track of our degrees for us with $k \geq 0$, we have, using the above notation:

\section{Theorem 6.1}

$$
\begin{aligned}
\pi_{*}(Y 0) \cong \operatorname{CORE} \times \prod^{k \geq 0} g^{-k}\left(\pi_{*} b o\langle 8\rangle \times \pi_{*} b o\langle 12\rangle \times \pi_{*} b o\langle 17\rangle \times \pi_{*} b o\langle 25\rangle\right. \\
\left.\times \pi_{*} b o\langle 32\rangle \times \pi_{*} b o\langle 34\rangle \times \pi_{*} b o\langle 42\rangle \times \pi_{*} b o\langle 48\rangle\right) .
\end{aligned}
$$

Notice that by Bott periodicity there are not so many different types as this seems to imply. The homotopy associated with $48 k+8,48 k+32$, and $48 k+48$ are just suspensions of the homotopy of bo. $48 k+12$ is just $b o\langle 4\rangle$, and $48 k+17$ and $48 k+25$ are $b o\langle 1\rangle$. Finally, $48 k+34$ and $48 k+42$ are associated with $b o\langle 2\rangle$.

We keep the notation as is though because $h$ maps these homotopy groups injectively following this sequence:

$$
\begin{array}{r}
\cdots \longrightarrow g^{-k} \pi_{*} b o\langle 48\rangle \longrightarrow g^{-k} \pi_{*} b o\langle 42\rangle \longrightarrow g^{-k} \pi_{*} b o\langle 34\rangle \longrightarrow \\
\longrightarrow g^{-k} \pi_{*} b o\langle 32\rangle \longrightarrow g^{-k_{*}} \longrightarrow o\langle 25\rangle \longrightarrow g^{-k} \pi_{*} b o\langle 17\rangle \longrightarrow \\
\longrightarrow g^{-k} \pi_{*} b o\langle 12\rangle \longrightarrow g^{-k} \pi_{*} b o\langle 8\rangle \longrightarrow g^{-k+1} \pi_{*} b o\langle 48\rangle \longrightarrow \\
\longrightarrow g^{-k+1} \pi_{*} b o\langle 42\rangle \longrightarrow g^{-k+1} \pi_{*} b o\langle 34\rangle \longrightarrow g^{-k+1} \pi_{*} b o\langle 32\rangle \longrightarrow \cdots
\end{array}
$$


In the formula for the homotopy of $Y 0$, we can replace CORE with $\mathrm{CORE}^{+}$and let $k \in \mathbb{Z}$ and we have the homotopy for all of $E R(2)$. The copies of $\mathbb{Z}_{(2)} \times B O$ are associated with $k<0$.

\section{$7 \quad E R(2)$ and homology}

In [8], the complete computation for the homology of all spaces in the Omega spectrum for $E R(2)$ is carried out. We would like to have the homology of the spaces $Y 0, Y 1$ and $Y 2$. These are easy to read off of the results of [8]. We need to review some notation.

We are only interested in the $0,-16$ and -32 spaces in the Omega spectrum. The dimension zero elements are free over $\mathbb{Z}_{(2)}$ on $\left[\alpha^{i}\right]$ and $\left[g^{k}\right]$ with $i \geq 0$ and $k \in \mathbb{Z}$.

We have elements $b_{2^{i}}=b_{(i)} \in H_{2^{i+1}} \underline{E}(2)_{2}$ that come from the complex projective space elements $b_{j}$. We have corresponding elements defined using the invertible element $y(2)$ :

$$
\beta_{2^{i}}=\beta_{(i)} \in H_{2^{i}} \underline{E R}(2)_{-16}=H_{2^{i}} \underline{\underline{E} \mathbb{R}}(2)_{1+\alpha} .
$$

The $\beta_{(i)}$ all come from the real projective space elements $\beta_{2^{i}}$.

Let $J=\left(j_{0}, j_{1}, \ldots\right)$ have $j_{i} \geq 0$ with only a finite number not equal to zero. Let $\Delta_{i}$ be the sequence with 1 in the $i$-th place and zeros everywhere else. We define

$$
\beta^{J}=\beta_{(0)}^{j_{0}} \beta_{(1)}^{j_{1}} \beta_{(2)}^{j_{2}} \cdots \text {. }
$$

Recall that in a Hopf ring we have two products, the circle product coming from the ring structure and the star product coming from the Hopf algebra structure. We suppress the circle from our notation so the above products are circle products. We define $\beta^{J}\left[\alpha^{i}\right]\left[g^{k}\right]$ to be allowable if all $j_{k}<2$ when $i>0$ and $J \neq 2 \Delta_{i_{1}}+4 \Delta_{i_{2}}+J^{\prime}$, $i_{1} \leq i_{2}$, when $i=0$ (where each $j_{k}^{\prime} \geq 0$ as usual). Define the length of $J$ to be $\ell(J)=\Sigma j_{i}$.

From $[8,(2.6)]$ we have (where $P$ denotes the polynomial algebra mod 2):

$$
H_{*} \underline{E R}(2)_{-16 *} \simeq P\left[\beta^{J}\left[\alpha^{i}\right]\left[g^{k}\right]\right], \quad \beta^{J}\left[\alpha^{i}\right]\left[g^{k}\right] \text { allowable. }
$$

We are only interested in the $0,-16$ and -32 spaces, and, using $h=\alpha^{3} g^{-1}$ we can rewrite this. Consider first the positive degree elements:

$$
H_{*} \underline{E R}(2)_{0} \simeq P\left[\beta^{J}\left[\alpha^{3-\epsilon}\right]\left[g^{-a-1}\right]\left[h^{s}\right]\right],
$$

where $\beta^{J}\left[\alpha^{3-\epsilon}\right]\left[g^{-a-1}\right]\left[h^{s}\right]$ is allowable and $\ell(J)=3 a+\epsilon, a \geq 0,0<\epsilon \leq 3, s \geq 0$. 
Whenever there is an $\alpha$, either because $s>0$ or $3-\epsilon>0$, we have $j_{i}<2$ from the definition of allowable. If $s>0$ we have exactly one element in each degree, giving us precisely the homology of the $B O$ associated with $h^{s}$. If $s=0$ we get the homology of one more $B O$ when using all of these $J$ with $j_{i}<2$. What is left must be the homology of $Y 0$, and that is:

$$
H_{*}(Y 0) \cong P\left[\beta^{J}\left[g^{-a}\right]\right], \quad J \text { allowable, } \ell(J)=3 a, \text { some } j_{i}>1 .
$$

Note that the lowest degree element is, indeed, in degree 3: $\beta_{(0)}^{3}\left[g^{-1}\right]$. A similar analysis gives:

$$
\begin{array}{ll}
H_{*}(Y 1) \cong P\left[\beta^{J}\left[g^{-a}\right]\right], & J \text { allowable, } \ell(J)=3 a+1, \text { some } j_{i}>1 . \\
H_{*}(Y 2) \cong P\left[\beta^{J}\left[g^{-a}\right]\right], & J \text { allowable, } \ell(J)=3 a+2, \text { some } j_{i}>1 .
\end{array}
$$

For $H_{*}(Y 1)$, the lowest degree element is in degree 4 and is $\beta_{(0)}^{4}\left[g^{-1}\right]$. For $H_{*}(Y 2)$, the lowest degree element is in degree 2, associated with $B \mathrm{SO}$, and is $\beta_{(0)}^{2}$, and the degree 5 element in $H_{*}\left(Y 2^{\prime}\right)$ (from the introduction) is $\beta_{(0)}^{5}\left[g^{-1}\right]$. Putting this all together, we get:

\section{Theorem 7.2}

$$
H_{*}(Y 0 \times Y 1 \times Y 2) \simeq P\left[\beta^{J}\left[g^{-a}\right]\right],
$$

where $J$ is allowable, $0 \leq \ell(J)-3 a<3$, some $j_{i}>1$.

\section{Appendix A}

For the reader's benefit, we will reprove some results of $\mathrm{Hu}$ [5] regarding the homotopy of $\mathbb{B P}\langle n\rangle$ in a manner that is helpful to us. Given a $\mathbb{Z} /(2)$-equivariant spectrum $\mathbb{E}$, we shall use the (nonstandard) notation $\widehat{\mathbb{E}}$ to denote the Borel cohomology spectrum $\operatorname{Map}\left(E \mathbb{Z} /(2)_{+}, \mathbb{E}\right)$. This notation is in keeping with [5], where the Borel cohomology spectrum of $\mathbb{E}$ is thought of as the completion of $\mathbb{E}$.

To perform our computation, the standard method used is to consider the Tate diagram given by rows that are cofibrations:

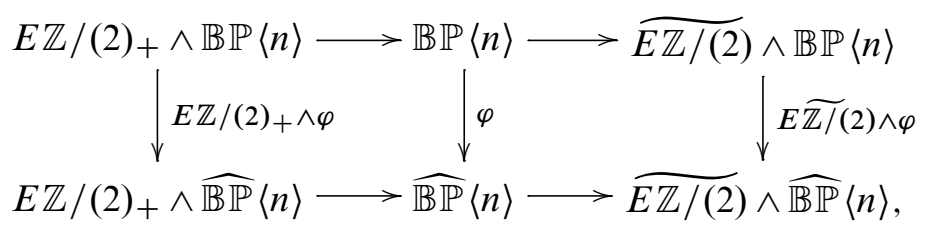

where $\varphi: \mathbb{B P}\langle n\rangle \rightarrow \widehat{\mathbb{B P}}\langle n\rangle$ is the "completion" map given by the canonical map $\varphi: \mathbb{B P}\langle n\rangle \rightarrow \operatorname{Map}\left(E \mathbb{Z} /(2)_{+}, \mathbb{B P}\langle n\rangle\right)$ induced by the projection $E \mathbb{Z} /(2)_{+} \rightarrow S^{0}$. 
Since the map $\varphi$ is a (nonequivariant) equivalence, it follows that $E \mathbb{Z} /(2)_{+} \wedge \varphi$ is an equivariant equivalence. Hence, the fiber of $\varphi$ is equivalent to the fiber of $\widetilde{E \mathbb{Z} /(2)} \wedge \varphi$. One has standard (trigraded) spectral sequences that compute the homotopy of the spectra

$$
\widehat{\mathbb{B P}}\langle n\rangle \text { and } \overparen{E \mathbb{Z} /(2)} \wedge \widehat{\mathbb{B P}}\langle n\rangle \text {, }
$$

called the Borel cohomology and the Tate cohomology spectral sequences for $\mathbb{B} \mathbb{P}\langle n\rangle$ respectively. The respective $E_{2}$-terms are given by:

$$
\begin{aligned}
& H^{p}\left(\mathbb{Z} /(2), \pi_{\mu} \mathbb{B} \mathbb{P}\langle n\rangle\right) \Longrightarrow \pi_{\mu-p} \widehat{\mathbb{B P}}\langle n\rangle, \\
& \widehat{H}^{p}\left(\mathbb{Z} /(2), \pi_{\mu} \mathbb{B} \mathbb{P}\langle n\rangle\right) \Longrightarrow \pi_{\mu-p} \widehat{E \mathbb{Z} /(2)} \wedge \widehat{\mathbb{B P}}\langle n\rangle,
\end{aligned}
$$

where $\widehat{H}(\mathbb{Z} /(2))$ denotes Tate cohomology and $\mu$ is any element in $R O(\mathbb{Z} /(2))$ that can be written as $a+b \alpha$, where $\alpha$ is the sign representation of $\mathbb{Z} /(2)$. One may even write the respective $E_{1}$ terms explicitly as:

$$
\mathbb{Z}_{(2)}\left[v_{i}, \sigma^{ \pm 1}, a\right] \quad \text { and } \quad \mathbb{Z}_{(2)}\left[v_{i}, \sigma^{ \pm 1}, a^{ \pm 1}\right], \quad i \leq n, v_{0}=2 .
$$

The classes $v_{i}, \sigma, a$ have tridegree given by:

$$
\left|v_{i}\right|=\left(0,\left(2^{i}-1\right)(1+\alpha)\right), \quad|\sigma|=(0,-1+\alpha), \quad|a|=(1,1-\alpha) .
$$

In addition, the classes $v_{i}$ and $a$ are permanent cycles, and the differentials are given by the universal differentials computed for the Borel cohomology spectral sequence for $\mathbb{M U}_{(2)}[6]$ :

$$
d_{2^{k+1-1}} \sigma^{-2^{k}}=v_{k} a^{2^{k+1}-1} .
$$

These differentials induce differentials in the Tate cohomology spectral sequence and it is straightforward to derive the following result in [5]:

Corollary A.1 The Tate spectral sequence for $\mathbb{B P}\langle n\rangle$ collapses at $E_{2^{n+1}-1}$ to yield:

$$
\pi_{*} \widehat{E \mathbb{Z} /(2)} \wedge \widehat{\mathbb{B P}}\langle n\rangle=\mathbb{Z} /(2)\left[\sigma^{ \pm 2^{n+1}}, a^{ \pm 1}\right] .
$$

In addition, multiplication by $v_{i}$ is given by zero in $\pi_{*} \widetilde{E \mathbb{Z} /(2)} \wedge \mathbb{B P}\langle n\rangle$. The image of the map

$$
\pi_{*} \widehat{\mathbb{B P}}\langle n\rangle \longrightarrow \pi_{*} \overparen{E \mathbb{Z} /(2)} \wedge \widehat{\mathbb{B P}}\langle n\rangle
$$

is given by $\mathbb{Z} /(2)\left[\sigma^{ \pm 2^{n+1}}, a\right]$ and the kernel of the map is contained in the image of $\pi_{*} \mathbb{M U}_{(2)}$.

The next step is to compute the homotopy of the geometric fixed-point spectrum $\widetilde{E \mathbb{Z} /(2)} \wedge \mathbb{B} \mathbb{P}\langle n\rangle$, and identify it as a subgroup of the homotopy of the Tate cohomology spectrum. Since there is no natural spectral sequence that computes the homotopy 
of the geometric fixed points, the argument used in [5] is by induction starting with $\mathbb{B} \mathbb{P}\langle 0\rangle$ and inducting upwards. Alternatively, one may start with the observation that

$$
\widetilde{E \mathbb{Z} /(2)} \wedge \mathbb{B} \mathbb{P}=H \mathbb{Z} /(2)\left[a^{ \pm 1}\right],
$$

and kill the elements $v_{i}$ for $i>n$. Then using the computations made in [4], we see that $\pi_{*} \widetilde{E \mathbb{Z} /(2)} \wedge \mathbb{B P}\langle n\rangle$ can be identified with the subgroup of the homotopy of the Tate cohomology spectrum given by $\mathbb{Z} /(2)\left[\sigma^{-2^{n+1}}, a^{ \pm 1}\right]$ as calculated in [5]. From the above observation we derive:

Corollary A.2 Let $F$ denote the fiber of the map from the geometric fixed-point spectrum to the Tate fixed-point spectrum. Then there is a short exact sequence in homotopy:

$$
0 \longrightarrow \pi_{*} \widetilde{E \mathbb{Z} /(2)} \wedge \mathbb{B P}\langle n\rangle \longrightarrow \pi_{*} \widetilde{E \mathbb{Z} /(2)} \wedge \widehat{\mathbb{B P}}\langle n\rangle \longrightarrow \pi_{*} \Sigma F \longrightarrow 0 .
$$

In particular, $\pi_{*} \Sigma F$ is isomorphic to the ideal generated by $\sigma^{2^{n+1}}$ in $\mathbb{Z} /(2)\left[\sigma^{2^{n+1}}, a^{ \pm 1}\right]$. In addition, multiplication by $v_{i}$ is given by zero on $\pi_{*} F$.

Now recall that the fiber of completion map $\varphi$ is given by $F$. Since we know the homotopy of $F$, and the fact that inverting $v_{n}$ collapses the fiber, we have essentially managed to show:

Theorem A.3 The map $\varphi$ is an equivalence on inverting $v_{n}$. In addition, the map $\pi_{a+b \alpha} \mathbb{B P}\langle n\rangle \rightarrow \pi_{a+b \alpha} \widehat{\mathbb{B P}}\langle n\rangle$ is an isomorphism for $a \geq b>-2^{n+1}$. Furthermore, in this range, the homotopy is given by the image of $\pi_{a+b \alpha} \mathbb{M U}(2)$.

\section{References}

[1] J M Boardman, D C Johnson, W S Wilson, Unstable operations in generalized cohomology, from: "Handbook of algebraic topology", (M James, I editor), North-Holland, Amsterdam (1995) 687-828 MR1361900

[2] J M Boardman, W S Wilson, Unstable splittings related to Brown-Peterson cohomology, from: "Cohomological methods in homotopy theory", (J Aguadé, C Broto, C Casacuberta, editors), Progr. Math. 196, Birkhäuser, Basel (2001) 35-45 MR1851246

[3] D M Davis, A strong nonimmersion theorem for real projective spaces, Ann. of Math. 120 (1984) 517-528 MR769162

[4] M A Hill, M J Hopkins, D C Ravenel, On the non-existence of elements of Kervaire invariant one arXiv:0908.3724

[5] P Hu, On Real-oriented Johnson-Wilson cohomology, Algebr. Geom. Topol. 2 (2002) 937-947 MR1936976 
[6] P Hu, I Kriz, Real-oriented homotopy theory and an analogue of the Adams-Novikov spectral sequence, Topology 40 (2001) 317-399 MR1808224

[7] N Kitchloo, W S Wilson, On fibrations related to real spectra, from: "Proceedings of the Nishida Fest", (M Ando, N Minami, J Morava, S Wilson, W editors), Geom. Topol. Monogr. 10, Geom. Topol. Publ., Coventry (2007) 237-244 MR2402788

[8] N Kitchloo, W S Wilson, On the Hopf ring for ER(n), Topology Appl. 154 (2007) 1608-1640 MR2317067

[9] N Kitchloo, W S Wilson, The second real Johnson-Wilson theory and nonimmersions of $R P^{n}$, Homology, Homotopy Appl. 10 (2008) 223-268 MR2475624

[10] N Kitchloo, W S Wilson, The second real Johnson-Wilson theory and nonimmersions of $R P^{n}$. II, Homology, Homotopy Appl. 10 (2008) 269-290 MR2475625

[11] M Mahowald, C Rezk, Topological modular forms of level 3, Pure Appl. Math. Q. 5 (2009) 853-872 MR2508904

[12] W S Wilson, The $\Omega$-spectrum for Brown-Peterson cohomology. II, Amer. J. Math. 97 (1975) 101-123 MR0383390

Department of Mathematics, Johns Hopkins University 3400 N. Charles St., Baltimore, MD 21218, USA

nitu@math.jhu.edu, wsw@math.jhu.edu

http://www.math.jhu.edu/ nitu/, http://www.math.jhu.edu/ wsw/

Received: 20 March 2012 Revised: 4 August 2012 